\title{
A pilot study of circulating PPAR- $\gamma$ receptor protein in elderly patients with atrial fibrillation
}

Qing Lin ${ }^{1}$, Lina Jia², Yanshu Sun ${ }^{1}$

1Department of Geriatrics, Peking University First Hospital, Beijing, China 2Department of Geriatrics, Beijing Shijitan Hospital, Beijing, China

\author{
Submitted: 12 April 2011 \\ Accepted: 3 November 2011
}

Arch Med Sci 2012; 8, 3: 471-476

DOI: 10.5114 /aoms.2012.29402

Copyright $\odot 2012$ Termedia \& Banach
Corresponding author:

Yanshu Sun MD

No. 8 Xishiku Street

West District

Beijing 100034, China

Phone/fax: 86-10-83572997

E-mail:

pingguo700@hotmail.com

\begin{abstract}
Introduction: The present study aimed to investigate the relationship between serum peroxisome proliferator-activated receptor- $\gamma$ (PPAR- $\gamma$ ) protein concentration and inflammatory markers in elderly patients with atrial fibrillation (AF). Material and methods: We enrolled a total of 73 elderly patients: 45 with AF as the test group and 28 in sinus rhythm as a control group. We assayed serum PPAR- $\gamma$ receptor protein, high-sensitivity C-reactive protein (hs-CRP), interleukin6 (IL-6), and tumour necrosis factor- $\alpha$ (TNF- $\alpha$ ). The study protocol and written informed consent were approved by the Ethics Committee of Clinical Research, Peking University First Hospital.

Results: The concentration of PPAR- $\gamma$ receptor protein was lower in AF patients than that in the control group ( $p<0.01$ ), and the concentrations of hs-CRP, IL-6, and TNF- $\alpha$ were higher than those in the control group (all $p<0.01$ ). The PPAR- $\gamma$ level was negatively correlated with hs-CRP, IL- 6 , and left atrium diameter (LAD) level (all $p<0.05)$. On logistic regression analysis, PPAR- $\gamma$, hs-CRP, TNF- $\alpha$ and LAD level were associated with AF.

Conclusions: Elderly patients with AF show an inflammatory state and atrial remodeling. The PPAR- $\gamma$ receptor protein concentration is inversely linked with inflammation in AF. As an important transcription factor regulating inflammatory gene expression, PPAR- $\gamma$ may take part in the pathogenesis of AF.
\end{abstract}

Key words: atrial fibrillation, peroxisome proliferator-activated receptor- $\gamma$, inflammation.

\section{Introduction}

Atrial fibrillation (AF) is a common arrhythmia which leads to substantial morbidity and mortality, as it is related to many cardiac diseases and increases the risk of stroke, coronary events, and congestive heart failure [1]. The prevalence and incidence of AF increase sharply with age and have been described as having reached epidemic proportions [2, 3]. The prevalence doubles with each decade of age, reaching almost $9 \%$ at age 80 to 89 years. Currently, approximately 2.3 million people in the United States are diagnosed with $\mathrm{AF}$, and this number is expected to increase to 5.6 million by 2050 . Thus, with the aging of the population, AF management will be an increasing problem for the medical and social care systems.

Although AF is the most prevalent arrhythmia, its underlying pathophysiological mechanisms are not fully understood. Recent evidence suggests inflammation may be important in the pathogenesis of AF and 
investigation of this and their potential for development of new effective treatment strategies for $\mathrm{AF}$ are of great interest [4-6]. Several cytokines are produced during AF and participate in the inflammatory process. C-reactive protein (CRP) levels can predict an association with AF and also future development of AF [6]. Furthermore, increased CRP levels are associated with increased risk of $A F$ recurrence after successful electrical cardioversion, and precardioversion CRP levels may provide prognostic information of the success of the procedure [7]. Sata et al. reported significantly higher high-sensitivity CRP (hs-CRP), interleukin-6 (IL-6) and tumor necrosis factor- $\alpha$ (TNF- $\alpha$ ) levels in patients with paroxysmal AF than in controls [8]. Histological studies have shown inflammatory infiltrates, myocyte necrosis, and fibrosis in atrial biopsies of patients with $\operatorname{AF}[9,10]$. Thus, although a causal relationship between the inflammatory process and AF remains unclear, inflammatory changes may contribute to atrial remodeling and persistence of $\mathrm{AF}$.

Peroxisome proliferator-activated receptor- $\gamma$ (PPAR- $\gamma$ ) is a ligand-dependent transcription factor belonging to the nuclear hormone receptor superfamily. It can heterodimerize with retinoid $X$ receptors, forming a complex that regulates gene expression. Ligand binding results in a conformational change in the receptor protein permitting corepressor dissociation and concomitant coactivator association. The heterodimer-coactivator complex binds to specific response elements in the promoter regions of target genes to regulate DNA transcription $[11,12]$. Various endogenous and synthetic PPAR- $\gamma$ ligands exist. Thiazolidinediones including rosiglitazone, pioglitazone, and troglitazone are highly potent synthetic PPAR- $\gamma$ agonists.

The actions of PPAR- $\gamma$ were originally thought to be limited to the control of lipid and glucose metabolism. However, accumulating evidence suggests that PPAR- $\gamma$ agonists act as novel anti-inflammatory agents by negatively regulating the expression of pro-inflammatory genes induced in response to inflammatory cell activation [13]. Several studies have examined the association between PPAR- $\gamma$ and cardiovascular disease [14]. However, few studies have investigated the role of PPAR- $\gamma$ in the pathogenesis of AF. Therefore, we aimed to assess the change in serum level of PPAR- $\gamma$ receptor protein and levels of inflammatory factors in elderly patients with $\mathrm{AF}$.

\section{Material and methods}

\section{Subjects}

We recruited 45 in-patients with AF from the Geriatric Department of Peking University First Hospital (Beijing, China) between July 2008 and July 2009. Twenty patients had paroxysmal AF and 25 had persistent AF. Paroxysmal AF self-terminates within 7 days of recognized onset. Persistent AF does not terminate spontaneously within 7 days and/or is terminated electrically or pharmacologically. Twentyeight age-matched patients in sinus rhythm were recruited as a control group. Exclusion criteria for this group were recent infection, hepatic or renal dysfunction, left ventricular ejection fraction $<45 \%$, malignant tumor, autoimmune disease, thyroid dysfunction, surgery or stroke within 3 months, acute coronary syndrome, and treatment with anti-inflammatory medications such as nonsteroidal anti-inflammatory drugs or glucocorticoids.

All participants underwent a comprehensive examination including standard medical history, physical examination, routine laboratory tests, and 12-lead electrocardiography. We evaluated valvular function, left ventricular size and function (ejection fraction), and left atrial diameter by transthoracic echocardiography. The diameter of the left atrium was measured in the parasternal long-axis view. The study protocol and written informed consent were approved by the Ethics Committee of Clinical Research, Peking University First Hospital.

\section{Blood sampling and laboratory tests}

Blood samples were obtained early in the day after an overnight fast, immediately transferred into a glass tube containing disodium EDTA, centrifuged for $20 \mathrm{~min}$ at $1,000 \mathrm{~g}$, separated in aliquots and then stored at $-80^{\circ} \mathrm{C}$. Staff blinded to clinical data performed measurements. Enzyme-linked immunosorbent assay kits were used to measure PPAR- $\gamma$ receptor protein (GBD, USA), IL-6 (SenXiong, Shanghai, China) and TNF- $\alpha$ (SenXiong, Shanghai, China) according to the manufacturers' instructions. The hs-CRP levels were measured by an immunonephelometry assay (First Chemical Pharmaceutical Co., Japan). The lower limits of detection were $0.1 \mathrm{pg} / \mathrm{ml}$ for PPAR- $\gamma, 0.1 \mathrm{mg} / \mathrm{l}$ for hs-CRP, and $8 \mathrm{pg} / \mathrm{ml}$ for IL- 6 and TNF- $\alpha$.

\section{Statistical analysis}

Data for normally distributed continuous variables are given as means \pm SD or number (\%) and non-normally distributed variables as medians with inter-quartile ranges. Student's t-test was used for comparisons of age, body mass index, blood pressure, blood lipid levels, fasting plasma glucose, and echocardiographic indicators. Comparisons of LAD were analyzed by ANOVA, and the Student-Newman-Keuls test. Categorical variables were analyzed by the $\chi^{2}$ test. Plasma levels of PPAR- $\gamma$ receptor protein and inflammatory factors were analyzed by Mann-Whitney test (2 groups) and Kruskal-Wallis test ( $n$ groups). Correlation was analyzed by Spearman's correlation method. The predictive significance of multiple variables was compared by stepwise lo- 
gistic regression analysis. A value of $p<0.05$ was considered statistically significant. All analyses were carried out using SPSS v13.0 (SPSS Inc., Chicago, IL, USA).

\section{Results}

\section{Baseline characteristics}

The patients' clinical characteristics and medications are listed in detail in Table I. Of the 45 patients with AF, 28 were 65 to 80 years old, and 17 were older than 80 years. The experimental and control groups did not differ in age, sex, body mass index, blood pressure, blood lipid levels, glucose, history of associated diseases, or medications. Patients with paroxysmal $A F$ were in sinus rhythm at the time of blood sampling. The left atrium diameter (LAD) in patients with AF was significantly larger than that in the control group (43.2 $\pm 6.1 \mathrm{~mm}$ vs. $36.6 \pm 3.4 \mathrm{~mm}$, $p<0.01)$. Furthermore, the LAD in patients with per- sistent AF was larger than that in the paroxysmal AF group (45.2 $\pm 6.0 \mathrm{~mm}$ vs. $40.8 \pm 5.4 \mathrm{~mm}, p<0.05)$.

\section{Expression of PPAR- $\gamma$, hs-CRP, IL-6, and TNF- $\alpha$ in serum}

As shown in Table II, serum levels of hs-CRP, IL-6, and TNF- $\alpha$ were higher in the AF group than in the control group (all $p<0.01$ ). The serum concentration of PPAR- $\gamma$ was lower in the AF group than in the control group $(p<0.01)$. Serum hs-CRP, IL-6, and TNF- $\alpha$ concentrations were significantly higher and PPAR- $\gamma$ concentration was significantly lower in patients with both paroxysmal and persistent AF than in controls (all $p<0.05$ ), with no difference between patients with persistent or paroxysmal AF in concentrations of these analyses (all $p>0.05$ ) (Figure 1). Patients 65 to 80 years old did not differ from those older than 80 in levels of PPAR- $\gamma$, hs-CRP, IL-6, or TNF- $\alpha$ (all $p>0.05$ ) (data not shown).

Table I. Baseline clinical characteristics of subjects

\begin{tabular}{|c|c|c|c|}
\hline Variables & Control group $(n=28)$ & AF group $(n=45)$ & Value of $p$ \\
\hline Age [years] & $74.8 \pm 7.4$ & $77.8 \pm 6.3$ & 0.071 \\
\hline Male, $n(\%)$ & $16(57.1)$ & $34(75.5)$ & 0.083 \\
\hline BMI $\left[\mathrm{kg} / \mathrm{m}^{2}\right]$ & $25.8 \pm 3.3$ & $24.2 \pm 3.3$ & 0.055 \\
\hline Systolic blood pressure [mm Hg] & $125.0 \pm 13.7$ & $123.7 \pm 11.3$ & 0.839 \\
\hline Diastolic blood pressure [mm Hg] & $70.0 \pm 8.9$ & $73.0 \pm 7.0$ & 0.434 \\
\hline Triglyceride [mmol/l] & $1.3 \pm 0.4$ & $1.0 \pm 0.2$ & 0.106 \\
\hline Total cholesterol [mmol/l] & $4.0 \pm 1.1$ & $3.6 \pm 0.7$ & 0.344 \\
\hline $\mathrm{HDL}-\mathrm{C}[\mathrm{mmol} / \mathrm{l}]$ & $0.9 \pm 0.2$ & $1.0 \pm 0.3$ & 0.532 \\
\hline LDL-C [mmol/l] & $2.3 \pm 0.8$ & $2.1 \pm 0.5$ & 0.462 \\
\hline $\mathrm{FPG}[\mathrm{mmol} / \mathrm{l}]$ & $5.0 \pm 0.5$ & $4.6 \pm 0.3$ & 0.145 \\
\hline \multicolumn{4}{|l|}{ Associated diseases } \\
\hline Hypertension, $n(\%)$ & $25(89.3)$ & $37(82.2)$ & 0.321 \\
\hline Coronary heart disease, $n(\%)$ & $15(53.6)$ & $27(60.0)$ & 0.382 \\
\hline Diabetes mellitus, $n(\%)$ & $11(39.3)$ & $10(22.2)$ & 0.097 \\
\hline Valvular heart disease, $n(\%)$ & $9(32.1)$ & $20(44.4)$ & 0.213 \\
\hline \multicolumn{4}{|l|}{ Echocardiography } \\
\hline $\operatorname{LAD}[\mathrm{mm}]$ & $36.6 \pm 3.4$ & $43.2 \pm 6.1$ & $<0.01$ \\
\hline LVDd [mm] & $48.7 \pm 3.8$ & $49.1 \pm 6.2$ & 0.777 \\
\hline LVEF [\%] & $59.9 \pm 6.5$ & $58.1 \pm 9.5$ & 0.388 \\
\hline \multicolumn{4}{|l|}{ Medications } \\
\hline CCB, $n(\%)$ & $12(42.9)$ & $21(46.7)$ & 0.470 \\
\hline$\beta$-Blockers, $n(\%)$ & $18(64.3)$ & $35(77.8)$ & 0.162 \\
\hline ACEI/ARB, $n(\%)$ & $19(67.9)$ & $29(64.4)$ & 0.485 \\
\hline Statins, $n(\%)$ & $15(53.6)$ & $27(60.0)$ & 0.382 \\
\hline
\end{tabular}

Data are expressed as mean $\pm S D ; B M I-$ body mass index, HDL-C-high-density lipoprotein cholesterol, LDL-C - low-density lipoprotein cholesterol, FPG -fasting plasma glucose, LAD - left atrial diameter, LVDd-end-diastolic left ventricle diameter, LVEF-left ventricular ejection fraction, $C C B$ - calcium channel blockers, ACEI - angiotensin-converting enzyme inhibitors, ARB - angiotensin receptor blocker 
Table II. Serum concentrations of peroxisome proliferator-activated receptor- $\gamma$ (PPAR- $\gamma$ ) and inflammatory factors in control and AF groups

\begin{tabular}{|lcc|}
\hline Variables & Control group $(n=28)$ & AF group $(n=45)$ \\
\hline PPAR- $\gamma[\mathrm{pg} / \mathrm{ml}]$ & 162.25 & 130.60 \\
& $(149.13,176.20)$ & $(102.35,153.90)^{*}$ \\
\hline hs-CRP $[\mathrm{mg} / \mathrm{l}]$ & 1.31 & 4.41 \\
& $(0.42,3.03)$ & $(1.23,9.15)^{\star}$ \\
\hline IL-6 [pg/ml] & 25.69 & 53.92 \\
& $(12.68,43.29)$ & $(42.50,70.02)^{*}$ \\
\hline TNF- $\alpha[\mathrm{pg} / \mathrm{ml}]$ & 184.69 & 282.92 \\
& $(152.71,251.36)$ & $(220.81,357.03)^{*}$ \\
\hline
\end{tabular}

Data are expressed as medians (interquartile range); *significant variation from control $(p<0.01)$; hs-CRP - high-sensitivity C-reactive protein, IL-6-interleukin-6, TNF- $\alpha$ - tumor necrosis factor- $\alpha$

The level of PPAR- $\gamma$ was negatively correlated with hs-CRP $(r=-0.267, p=0.023)$, IL-6 $(r=-0.324$, $p=0.005)$ and LAD in the parasternal long-axis view $(r=-0.312, p=0.007)$.
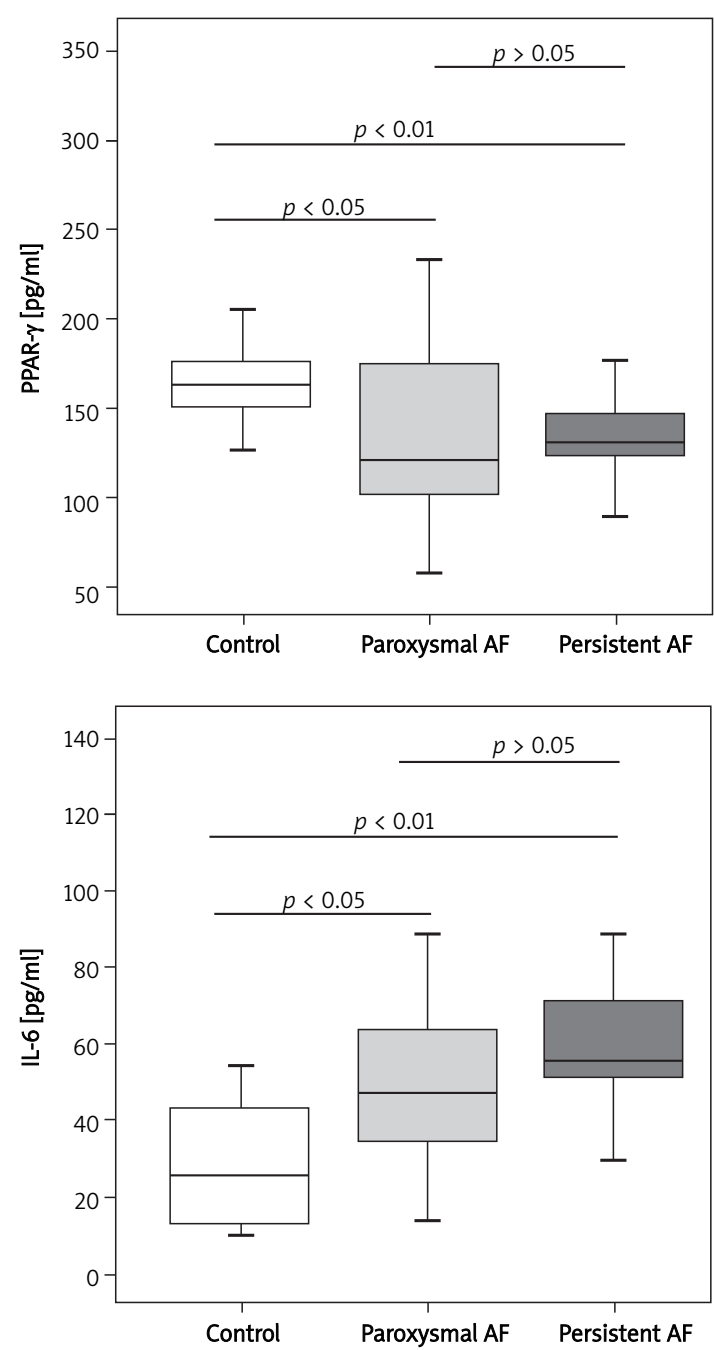

Table III. Logistic regression analysis of risk factors of atrial fibrillation

\begin{tabular}{|lccc|}
\hline Variables & Odds ratio & $\begin{array}{c}95 \% \text { Confidence } \\
\text { interval }\end{array}$ & Value of $p$ \\
\hline PPAR- $\gamma$ & 0.968 & $0.941-0.997$ & 0.029 \\
\hline hs-CRP & 1.431 & $1.073-1.907$ & 0.015 \\
\hline TNF- $\alpha$ & 1.016 & $1.002-1.030$ & 0.024 \\
\hline LAD & 1.372 & $1.100-1.710$ & 0.005 \\
\hline
\end{tabular}

PPAR- $\gamma$ - peroxisome proliferator-activated receptor- $\gamma$, hs-CRP-highsensitivity C-reactive protein, TNF- $\alpha$-tumor necrosis factor- $\alpha$, LAD - left atrium diameter

Multi-factor analysis results suggested that PPAR- $\gamma$, hs-CRP, TNF- $\alpha$, and LAD were all independent predictors of AF (Table III).

\section{Discussion}

The AF is the most common cardiac arrhythmia, and its prevalence increases with age. It is uncom-
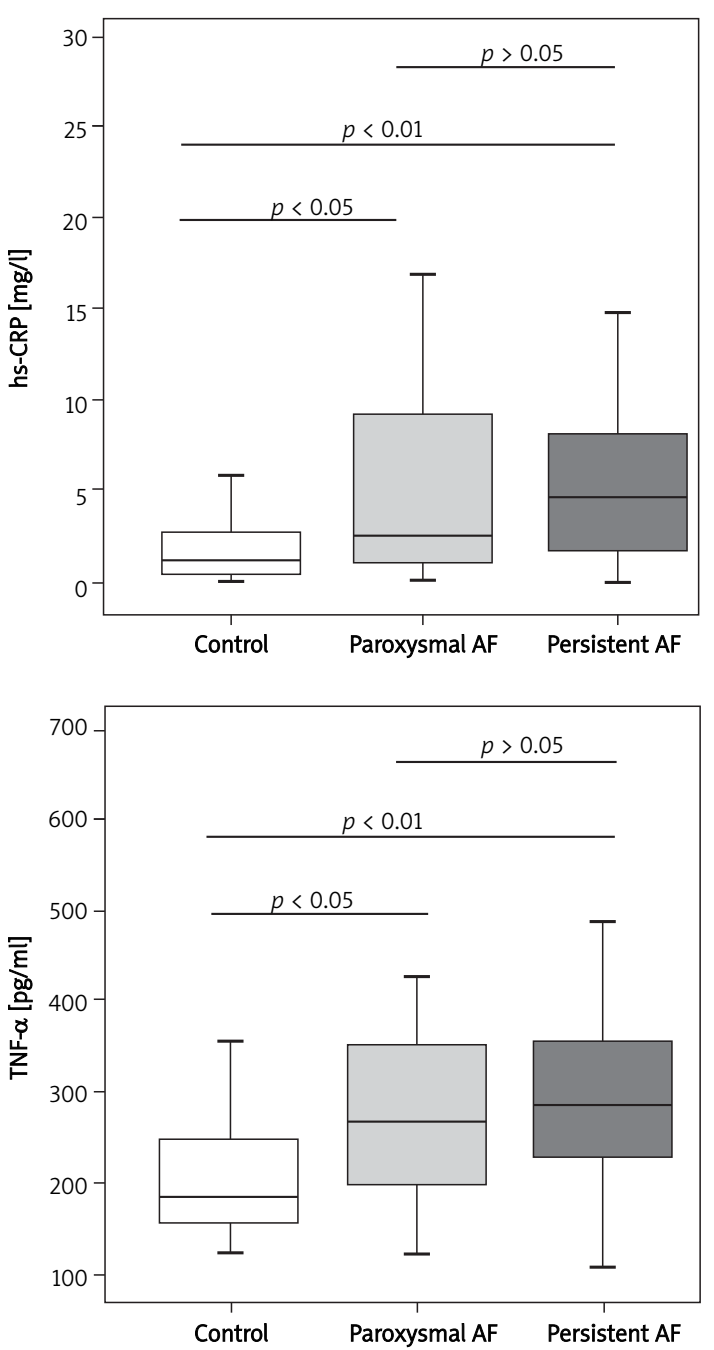

Figure 1. Serum levels of PPAR- $\gamma$ and inflammatory factors in controls and patients with paroxysmal and persistent AF. Box plots demonstrate median, and $25^{\text {th }}$ and $75^{\text {th }}$ percentile values 
mon for those under 60 years of age, but its prevalence increases markedly thereafter, affecting about $10 \%$ of the population by 80 years of age. Approximately one-third of all patients with AF are 80 years or older, and by 2050, half of the patients with AF will be in this age group [2]. Therefore, AF in elderly patients is a concern. Many studies have suggested that AF is associated with systemic inflammation. The PPAR- $\gamma$ agonists have demonstrated anti-inflammatory effects and inflammation is implicated in many cardiovascular diseases.

We investigated the relationship between serum PPAR- $\gamma$ receptor protein concentration and inflammatory factors in elderly patients with AF. The concentration of hs-CRP, IL- 6 , and TNF- $\alpha$ in peripheral blood was increased in all patients with AF regardless of AF type, paroxysmal or persistent. This has been previously described. The novelty of our hypothesis is that PPAR- $\gamma$ receptor protein might be a circulating inflammatory marker in AF. The notion seems far-fetched, since the other markers, CRP, IL-6, and TNF, function in the process of innate immunity and act locally to combat invaders, whilst PPAR- $\gamma$ functions as a regulator of transcription and is not known to be an inverse acute phase reactant: To our knowledge, no other publications have suggested this possibility.

The PPAR- $\gamma$ activation influences expression of genes involved in carbohydrate and lipid metabolism. Recent evidence suggests that activators of PPAR- $\gamma$ exert anti-inflammatory, anti-oxidative and anti-proliferative effects on vascular wall cells, thus decreasing the risk of atherosclerosis [15]. In patients with newly diagnosed type 2 diabetes and obesity, the PPAR- $\gamma$ agonist rosiglitazone significantly reduced serum concentrations of hs-CRP, IL- $1 \beta$, IL- 6 , TNF- $\alpha$, fasting plasma glucose and insulin resistance index [16]. In non-diabetic subjects with cardiovascular disease and elevated hs-CRP level, the PPAR- $\gamma$ agonist pioglitazone has anti-inflammatory effects as shown by decreased hs-CRP, matrix metalloproteinase-9, and plasminogen activator inhibitor-1 concentration [17]. These findings suggest that the PPAR- $\gamma$ system plays an anti-inflammatory role in a broad spectrum of cardiovascular diseases associated with AF, such as coronary artery disease, insulin resistance, type 2 diabetes mellitus, and hypertension. However, the role of PPAR- $\gamma$ in the pathogenesis of AF is untested.

We have demonstrated that the PPAR- $\gamma$ receptor protein concentration is decreased in patients with AF as compared with controls, regardless of AF type, paroxysmal or persistent. At the same time, in our study, PPAR- $\gamma$ receptor protein concentration was negatively correlated with hs-CRP and IL- 6 concentrations. Subsequent multivariate analysis indicated PPAR- $\gamma$ as an independent predictor of AF. Our findings are in agreement with Chen et al., who demon- strated that PPAR- $\gamma$ mRNA expression was decreased in AF patients with hypertension as compared with controls, while it was lower in the persistent than in the paroxysmal AF patients [18].

The development of AF leads to structural and electrical changes in the atria, known as remodeling. These changes further perpetuate the existence and maintenance of arrhythmia. Atrial remodeling represents a prominent mechanism of AF development and perpetuation, and inflammation and oxidative stress may be implicated in this process [19]. Our patients with AF had enlarged LAD (an index of atrial structural remodeling) as compared with controls; PPAR- $\gamma$ receptor protein concentration was negatively correlated with LAD in patients with AF. The association between inflammatory factors, PPAR- $\gamma$ and increased LAD supports a link between the burden of $A F$, inflammation, and atrial structural remodeling.

A few studies have suggested that PPAR- $\gamma$ activation might have favorable effects on AF. Kume et al. found that pioglitazone attenuates inflammatory atrial fibrosis and vulnerability to atrial fibrillation induced by pressure overload in rats [20]. Shimano et al. found that pioglitazone suppressed arrhythmogenic atrial structural remodeling and $\mathrm{AF}$ promotion in a rabbit model of congestive heart failure, and suggested that PPAR- $\gamma$ agonists may be a potential therapeutic agent for human AF [21]. Furthermore, Gu et al. observed that pioglitazone improved the preservation of sinus rhythm and reduced the reablation rate in patients with paroxysmal atrial fibrillation and type 2 diabetes mellitus after catheter ablation [22]. These results suggest that PPAR- $\gamma$ might have an active role in the generation, maintenance, and perpetuation of AF.

This study has several limitations. Our sample size, although small, was sufficient to show differences between controls and the AF group, but further studies with larger cohorts are needed to verify these results. In addition, medications such as renin-angiotensin system inhibitors were used in some patients. Although the medication use did not differ between patients with and without AF, these drugs may affect levels of inflammatory markers in patients with underlying cardiovascular disease. Finally, we did not evaluate the effects of AF duration on the association of inflammation and $A F$.

In conclusion, our study demonstrated lower serum concentrations of PPAR- $\gamma$ receptor protein and higher concentrations of hs-CRP, TNF- $\alpha$ and IL- 6 in patients with $A F$ than in those with sinus rhythm. As an important transcription factor regulating inflammatory gene expression, PPAR- $\gamma$ may take part in the pathogenesis of AF. Further studies are needed to elucidate the exact role of PPAR- $\gamma$ and to clarify the impact of PPAR- $\gamma$ agonist intervention in AF. 


\section{References}

1. Aksnes TA, Kjeldsen SE, Julius S. Atrial fibrillation and hypertension. Arch Med Sci 2009; 5: S267-72.

2. Kannel WB, Benjamin EJ. Current perceptions of the epidemiology of atrial fibrillation. Cardiol Clin 2009; 27: 13-24, vii.

3. Banach M, Misztal M, Goch A, Rysz J, Goch JH. Predictors of atrial fibrillation in patients following isolated surgical revascularization. A metaanalysis of 9 studies with 28786 patients. Arch Med Sci 2007; 3: 229-39.

4. Kourliouros A, Savelieva I, Kiotsekoglou A, Jahangiri M, Camm J. Current concepts in the pathogenesis of atrial fibrillation. Am Heart J 2009; 157: 243-52.

5. Li J, Solus J, Chen Q, et al. Role of inflammation and oxidative stress in atrial fibrillation. Heart Rhythm 2010; 7 : 438-44.

6. Rosiak M, Dziuba M, Chudzik M, et al. Risk factors for atrial fibrillation: not always severe heart disease, not always so 'lonely'. Cardiol J 2010; 17: 437-42.

7. Liu T, Li G, Li L, Korantzopoulos P. Association between C-reactive protein and recurrence of atrial fibrillation after successful electrical cardioversion: a meta-analysis. J Am Coll Cardiol 2007; 49: 1642-8.

8. Sata N, Hamada N, Horinouchi T, et al. C-reactive protein and atrial fibrillation. Is inflammation a consequence or a cause of atrial fibrillation? Jpn Heart J 2004; 45: 441-5.

9. Nguyen BL, Fishbein MC, Chen LS, Chen PS, Masroor S. Histopathological substrate for chronic atrial fibrillation in humans. Heart Rhythm 2009; 6: 454-60.

10. Qu YC, Du YM, Wu SL, Chen QX, Wu HL, Zhou SF. Activated nuclear factor-kappaB and increased tumor necrosis factor-alpha in atrial tissue of atrial fibrillation. Scand Cardiovasc J 2009; 43: 292-7.

11. Berger J, Moller DE. The mechanisms of action of PPARs. Annu Rev Med 2002; 53: 409-35.

12. Zieleniak A, Wójcik M, Woźniak LA. Structure and physiological functions of the human peroxisome proliferatoractivated receptor gamma. Arch Immunol Ther Exp (Warsz) 2008; 56: 331-45.

13. Straus DS, Glass CK. Anti-inflammatory actions of PPAR ligands: new insights on cellular and molecular mechanisms. Trends Immunol 2007; 28: 551-8.

14. Robinson E, Grieve DJ. Significance of peroxisome proliferator-activated receptors in the cardiovascular system in health and disease. Pharmacol Ther 2009; 122: 246-63.

15. Wang N, Yin R, Liu Y, Mao G, Xi F. Role of peroxisome proliferator-activated receptor-gamma in atherosclerosis: an update. Circ J 2011; 75: 528-5.

16. Zhu HL, Yu RM, Huang XZ, Huang W. Effects of rosiglitazone on inflammatory reaction and insulin resistance in obese patients with newly diagnosed type 2 diabetes. Nan Fang Yi Ke Da Xue Xue Bao 2008; 28: 1050-1.

17. Hanefeld M, Marx N, Pfützner A, et al. Anti-inflammatory effects of pioglitazone and/or simvastatin in high cardiovascular risk patients with elevated high sensitivity Creactive protein: the PIOSTAT Study. J Am Coll Cardiol 2007; 49: 290-7.

18. Chen X, Bing Z, He J, et al. Downregulation of peroxisome proliferator-activated receptor-gamma expression in hypertensive atrial fibrillation. Clin Cardiol 2009; 32: 337-45.

19. Aldhoon B, Melenovský V, Peichl P, Kautzner J. New insights into mechanisms of atrial fibrillation. Physiol Res 2010; 59: 1-12.

20. Kume O, Takahashi N, Wakisaka O, et al. Pioglitazone attenuates inflammatory atrial fibrosis and vulnerability to atrial fibrillation induced by pressure overload in rats. Heart Rhythm 2011; 8: 278-85.
21. Shimano M, Tsuji Y, Inden Y, et al Pioglitazone, a peroxisome proliferator-activated receptor-gamma activator, attenuates atrial fibrosis and atrial fibrillation promotion in rabbits with congestive heart failure. Heart Rhythm 2008; 5: 451-9.

22. Gu J, Liu X, Wang X, et al. Beneficial effect of pioglitazone on the outcome of catheter ablation in patients with paroxysmal atrial fibrillation and type 2 diabetes mellitus. Europace 2011; 13: 1256-61. 